\title{
A!
}

This is an electronic reprint of the original article.

This reprint may differ from the original in pagination and typographic detail.

Manzano, Gonzalo; Subero, Diego; Maillet, Olivier; Fazio, Rosario; Pekola, Jukka P.; Roldan, Edgar

\section{Thermodynamics of Gambling Demons}

Published in:

Physical Review Letters

DOI:

10.1103/PhysRevLett.126.080603

Published: 26/02/2021

\section{Document Version}

Publisher's PDF, also known as Version of record

Please cite the original version:

Manzano, G., Subero, D., Maillet, O., Fazio, R., Pekola, J. P., \& Roldan, E. (2021). Thermodynamics of Gambling Demons. Physical Review Letters, 126(8), [080603]. https://doi.org/10.1103/PhysRevLett.126.080603

This material is protected by copyright and other intellectual property rights, and duplication or sale of all or part of any of the repository collections is not permitted, except that material may be duplicated by you for your research use or educational purposes in electronic or print form. You must obtain permission for any other use. Electronic or print copies may not be offered, whether for sale or otherwise to anyone who is not an authorised user. 


\title{
Thermodynamics of Gambling Demons
}

\author{
Gonzalo Manzano $\odot,{ }^{1,2, *}$ Diego Subero $\odot,{ }^{3}$ Olivier Maillet $\odot,{ }^{3}$ Rosario Fazio, ${ }^{1,4}$ Jukka P. Pekola $\odot,{ }^{3}$ and Édgar Roldán $\oplus^{1, \dagger}$ \\ ${ }^{1}$ International Centre for Theoretical Physics ICTP, Strada Costiera 11, I-34151 Trieste, Italy \\ ${ }^{2}$ Institute for Quantum Optics and Quantum Information (IQOQI), Austrian Academy of Sciences, \\ Boltzmanngasse 3, 1090 Vienna, Austria \\ ${ }^{3}$ PICO group, QTF Centre of Excellence, Department of Applied Physics, Aalto University, 00076 Aalto, Finland \\ ${ }^{4}$ Dipartimento di Fisica, Università di Napoli "Federico II," Monte S. Angelo, I-80126 Napoli, Italy
}

(Received 4 September 2020; revised 23 November 2020; accepted 28 January 2021; published 26 February 2021)

\begin{abstract}
We introduce and realize demons that follow a customary gambling strategy to stop a nonequilibrium process at stochastic times. We derive second-law-like inequalities for the average work done in the presence of gambling, and universal stopping-time fluctuation relations for classical and quantum nonstationary stochastic processes. We test experimentally our results in a single-electron box, where an electrostatic potential drives the dynamics of individual electrons tunneling into a metallic island. We also discuss the role of coherence in gambling demons measuring quantum jump trajectories.
\end{abstract}

DOI: 10.1103/PhysRevLett.126.080603

Maxwell's demon, as introduced in 1867 [1], is a little intelligent being who acquires information about the microscopic degrees of freedom of two gases held in two containers at different temperatures, and separated by a rigid wall. The demon is able to control a tiny door, which can be opened at stochastic times, allowing fast particles from the cold container pass to the hotter one, and hence generating a heat current against a temperature gradient. This paradoxical behavior challenging the second law of thermodynamics, has its roots in the link between information and thermodynamics, which has fascinated scientists from more than a century [2]. Maxwell's demon is nowadays considered a paradigmatic example of feedback control, for which modified thermodynamic laws apply [3-6] which have been tested experimentally in classical [7-9] and quantum systems [10,11].

Here, we propose and realize a "gambling demon" which can be seen as a variant of the original Maxwell's thought experiment (Fig. 1). Such gambling demon invests work by performing a nonequilibrium thermodynamic process and acquires information about the response of the system during its evolution. Based on that information, the demon decides whether to stop the process or not following a given set of stopping rules and, as a result, may recover more work from the system than what was invested. However, differently to Maxwell's demon, a gambling demon does not control the system's dynamics, hence excluding the possibility of proper feedback control. This is analogous to a gambler who invests coins in a slot machine hoping to obtain a positive payoff. Depending on the sequence of outputs from the slot machine, the gambler may decide to either continue playing or stop the game (e.g., to avoid major losses), according to some prescribed strategy. How much work may the gambling demon save or extract on average in a given transformation by implementing a prescribed gambling strategy?

In this Letter, we derive and test experimentally universal equalities and inequalities for the work and entropy production fluctuations in Markovian nonequilibrium processes subject to gambling strategies that stop the process at a finite time during an arbitrary deterministic driving protocol. Our results apply to both classical and quantum

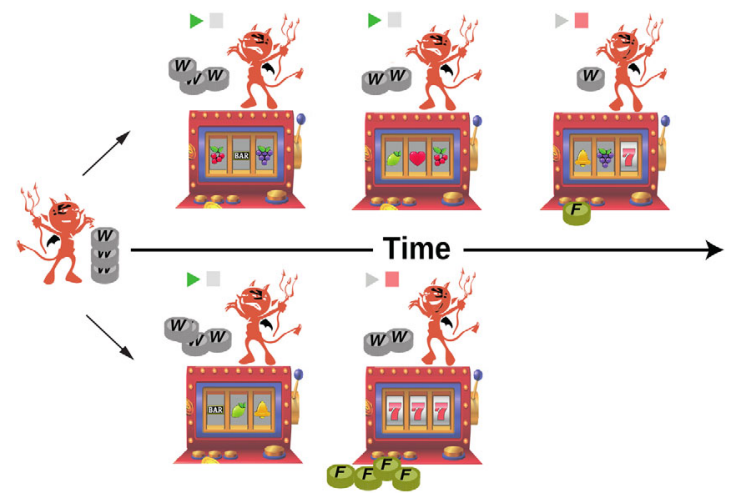

FIG. 1. Illustration of a gambling demon. The demon spends work ( $W$, silver coins) on a physical system (slot machine) hoping to collect free energy ( $F$, gold coins) by executing a gambling strategy. In each time step, the demon does work on the system (introduces a coin in the machine) and decides whether to continue ("play" sign) or to quit gambling and collect the prize ("stop" sign) at a stochastic time $\mathcal{T}$ following a prescribed strategy. In the illustration, the demon plays the slot machine until a fixed time $\mathcal{T}=3$ (top row) unless the outcome of the game is beneficial at a previous time, e.g., $\mathcal{T}=2$ (bottom row). Under specific gambling schemes, the demon can extract on average more free energy than the work spent over many iterations, a scenario that is forbidden by the standard second-law inequality. 
stochastic dynamics, and provide tight bounds to work extraction beyond the generalized second laws with continuous feedback control [9]. We derive these results applying the theory of Martingale stochastic processes. Martingales have been fruitfully applied in probability theory [12], quantitative finance [13], and more recently in nonequilibrium thermodynamics [14-18], providing insights beyond standard fluctuation theorems, e.g., universal bounds for the extrema and stopping-time statistics of thermodynamic quantities [14,15,19-22].

Work fluctuation theorems at stopping times.-We consider thermodynamic systems in contact with a thermal bath with inverse temperature $\beta=1 / k_{B} T$. The Hamiltonian $H$ of the system depends on time through an external control parameter $\lambda(t)$ following a prescribed deterministic protocol $\Lambda=\{\lambda(t) ; 0 \leq t \leq \tau\}$ of fixed duration $\tau$. The evolution of the system is subject to thermal fluctuations and thus we will describe its energetics using the framework of stochastic thermodynamics [23-25]. We denote the state (continuous or discrete) of the system at time $0 \leq t \leq \tau$ by $x(t)$, and the probability of observing a given trajectory $x_{[0, \tau]} \equiv\{x(t)\}_{t=0}^{\tau}$ associated with the driving protocol $\Lambda$ by $P\left(x_{[0, \tau]}\right)$. We assume its dynamics is stochastic and Markovian with probability density $\varrho(x, t)$. Thermodynamic variables such as system's energy $E(t)=H(x(t), \lambda)$ and entropy $S(t) \equiv$ $-k_{\mathrm{B}} \ln \varrho(x(t), t)$ are then stochastic processes, functionals of the stochastic trajectories $x_{[0, \tau]}$. We denote $W(\tau) \equiv$ $\int_{0}^{\tau} d t \partial_{t} H(x(t), t)$ the work exerted on the system up to time $\tau$, and $\Delta F(\tau) \equiv F(\tau)-F(0)$ the nonequilibrium free energy change, with $F(\tau) \equiv E(\tau)-T S(\tau)$. A key result from stochastic thermodynamics is the fluctuation theorem $\left\langle e^{-\beta(W-\Delta F)}\right\rangle=1[26,27]$, which implies the second-law inequality $\langle W\rangle-\langle\Delta F\rangle \geq 0$, where the averages $\langle\cdot\rangle$ are done over all possible trajectories of duration $\tau$ in the nonequilibrium protocol $\Lambda$.

We now ask ourselves whether the work fluctuation theorem and the second law still hold when averaging over trajectories stopped at stochastic times, following a custom "gambling" strategy. We consider strategies defined through a generic stopping condition that can be checked at any instant of time $t$ based only on the information collected about the system up to that time. In each run, the demon gambles applying the prescribed stopping condition, and decides whether to stop gambling or not depending on the system's evolution. In this work, we consider stopping times obeying $\mathcal{T}\left(x_{[0, \tau]}\right) \leq \tau$ for any trajectory $x_{[0, \tau]}$, i.e., demons which are enforced to gamble before or at the end of the nonequilibrium driving. For this class of systems we derive the inequality

$$
\langle W\rangle_{\mathcal{T}}-\langle\Delta F\rangle_{\mathcal{T}} \geq-k_{B} T\langle\delta\rangle_{\mathcal{T}},
$$

which involves averages of functionals of trajectories evaluated at stopping times $\langle O\rangle_{\mathcal{T}}=\sum_{x_{[0, \mathcal{T}}} P\left(x_{[0, \mathcal{T}]}\right) O(\mathcal{T})$, i.e., taken over many trajectories $x_{[0, \mathcal{T}]}$, each stopped at a stochastic time $\mathcal{T}$. Importantly, the quantity

$$
\delta(\mathcal{T}) \equiv \ln \left[\frac{\varrho(x(\mathcal{T}), \mathcal{T})}{\tilde{\varrho}(x(\mathcal{T}), \tau-\mathcal{T})}\right]
$$

denoted here as stochastic distinguishability, is a trajectorydependent measure of how distinguishable is $\varrho(x, \mathcal{T})$ with respect to the probability distribution $\tilde{\varrho}(x, \tau-\mathcal{T})$ at the same stopping (i.e., stochastic) time $\tau-\mathcal{T}$ in a reference timereversed process which is defined as follows. Its driving protocol $\tilde{\Lambda}=\{\tilde{\lambda}(\tau-t) ; 0 \leq t \leq \tau\}$ is the time-reversed picture of the forward protocol and its initial distribution is the distribution obtained at the end of the forward protocol, i.e., $\tilde{\varrho}(x, 0) \equiv \varrho(\tilde{x}, \tau)$ [28]. We derive Eq. (1) by extending the Martingale theory of stochastic thermodynamics to generic driven Markovian processes starting in arbitrary nonequilibrium conditions. This leads us to the fluctuation relation at stopping times

$$
\left\langle e^{-\beta(W-\Delta F)-\delta}\right\rangle_{\mathcal{T}}=1,
$$

which implies Eq. (1) by Jensen's inequality [29]. For the particular case of deterministic stopping at the end of the protocol $\mathcal{T} \rightarrow \tau$, we get $\delta(\mathcal{T}) \rightarrow 0$ and thus Eqs. (1) and (3) recover, respectively, the standard second law and the work fluctuation theorem, as expected.

Equation (1) reveals that the time asymmetry introduced by the driving protocol $\langle\delta\rangle_{\mathcal{T}} \geq 0$ enables for an apparent "second-law violation" i.e., $\langle W\rangle_{\mathcal{T}} \leq\langle\Delta F\rangle_{\mathcal{T}}$ at stopping times [42]. Because the system's evolution is stopped at stochastic times at which the external protocol takes on different values, the average work done in the gambling process is not bounded by the free energy change $\langle\Delta F\rangle_{\mathcal{T}}$ between the initial and the final state that one could reach with a deterministic protocol leading to the distribution $\varrho(x, \mathcal{T})$. The maximum extent of the violation of the traditional statement of the second law increases with $\langle\delta\rangle_{\mathcal{T}}$ i.e., when the process is driven far from equilibrium and the dynamics is strongly time asymmetric. Equations (1) and (3) are valid for any stopping strategy, thereby introducing a new level of universality. We next put to the test our results applying one specific set of stopping times to experimental data.

Experimental verification.-The experimental setup that we used to test the aforementioned predictions [shown in Fig. 2(a)] consists of two capacitively coupled metallic islands with small capacitance forming a single-electron transistor (SET) as a detector, and a single-electron box (SEB) as the system $[43,44]$. The SEB, with capacitance $C$, is left unbiased: the offset charge $n_{g}$ of the SEB can be externally tuned with a gate voltage $V_{g, \text { sys }}=e n_{g} / C_{g}$, where $C_{g}$ is the gate capacitance and $e$ the elementary charge. At low temperature $k_{B} T<e^{2} / 2 C$ the box can be approximated as a two-state system with charge number states $n=0$ and $n=1$, and the offset charge tuning enables the control of individual electrons on the island through the change in its electrostatic energy $E_{c}\left(n-n_{g}\right)^{2}$, with $E_{c}=1.94 k_{B} T$ and $T=0.67 \mathrm{~K}$. The other SET is used 

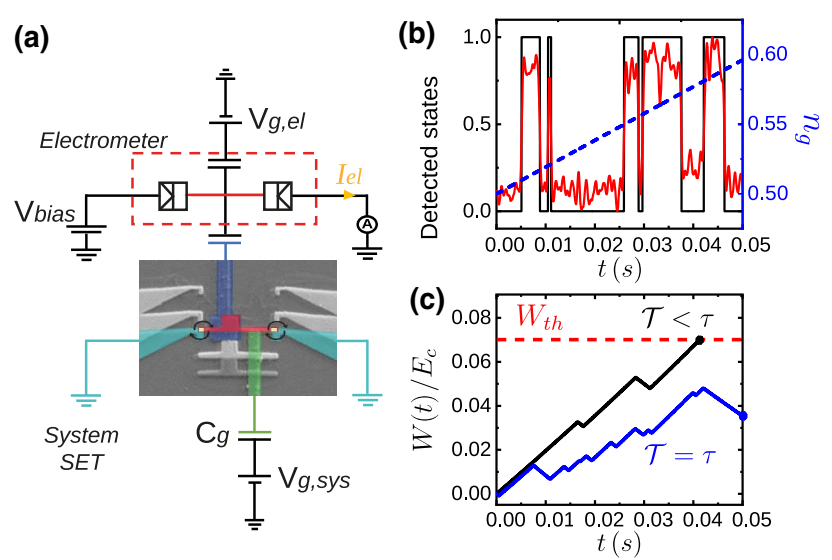

FIG. 2. (a) Scanning electron micrograph of the single-electron box (SEB) with false-color highlight on the $\mathrm{Cu}$ island (red) and the $\mathrm{Al}$ superconducting lead (turquoise). The superconducting leads are tunnel coupled through thin oxide barriers (yellow) to the island. The dc SET electrometer is coupled capacitively to the box through a bottom electrode (blue) which detects the excess charge of the box $n(t)$. (b) Representative time traces of the current measured through the electrometer (red solid line) and its digitized version (black solid line). The blue dashed line corresponds to the driving protocol $n_{g}(t)$ of duration $\tau=0.05 \mathrm{~s}$. (c) Example traces of the stochastic work done on the box as a function of time. We execute the following gambling strategy: the process is stopped at $\mathcal{T}<\tau$ (black line) only when the work reaches a threshold value $W_{\text {th }}$ (red dashed line) before $\tau$. On the contrary, the process is stopped at final protocol time $\mathcal{T}=\tau$ if the work threshold is never reached during the driving protocol (blue line).

as an electrometer biased with a low voltage: through capacitive coupling to the box, its output current is sensitive to the box charge state, taking two values corresponding to the system states. The tunneling of an electron into the island corresponds to a jump between the states $n=0$ and $n=1$ and is associated with an energy cost $\epsilon\left(n_{g}\right)=E_{c}\left(1-2 n_{g}\right)$. Through continuous monitoring of the box state $n(t)$ [see Fig. 2(b)], we experimentally evaluate at real time the heat exchange between the system and the bath during a driving protocol of the gate voltage $n_{g}(t)=\lambda(t)$. The tunneling (i.e., heat exchange) events occur at rates of order $\Gamma_{d} \sim 230 \mathrm{~Hz}$. If a jump occurs at time $t$ within a sampling time $\Delta t=20 \mu \mathrm{s} \ll \Gamma_{d}^{-1}$ at gate voltage $n_{g}$, the work increment is $\delta W=0$ and the heat increment is $\delta Q=\epsilon\left(n_{g}\right)\left[\delta Q=-\epsilon\left(n_{g}\right)\right]$ for an electron tunneling into (out) of the island. Conversely, if no jump occurs, $\delta Q=0$ and $\delta W=2 E_{c}\left(n_{g}-n\right) \dot{n}_{g} \delta t$.

The experimental driving protocol $\Lambda$ of duration $\tau$ is depicted in Fig. 2(b). The system is initially prepared at charge degeneracy, i.e., $n_{g}(0)=1 / 2$ at thermal equilibrium where the initial energies of states are equal, following a uniform distribution. Then the energy splitting is tuned according to a linear ramp, $n_{g}(t)=1 / 2+\Delta n_{g} t / \tau$, with $\Delta n_{g}=0.1$ fixed throughout the experiment. The protocol is repeated several times $(\sim 500-1000)$ to acquire sufficient statistics. The gambling strategy that we chose consists of stopping the dynamics at stochastic times $\mathcal{T}$ when the work exceeds a threshold value $W_{\text {th }}$ (red dashed line) or at $\tau$ otherwise. The gambling strategy was applied a posteriori on the data: for the same set of traces taken for the full protocol duration, the stopping condition (threshold work $W_{\text {th }}$ ) was varied between $10^{-4} E_{c}$ and $10^{-1} E_{c}$. In Fig. 2(c) we present two examples of stopped work trajectories where one reaches the threshold value at a time $\mathcal{T}<\tau$ (black line), while the other remains below the threshold until the final time $\tau$ (blue line).

Experimental values of $\langle W\rangle_{\mathcal{T}}-\langle\Delta F\rangle_{\mathcal{T}}$ and $-k_{B} T\langle\delta\rangle_{\mathcal{T}}$ are shown in Figs. 3(a) and 3(d) for two different ramps of durations $\tau=0.05 \mathrm{~s}$ (a) and $\tau=0.2 \mathrm{~s}$ (d) as a function of the work threshold $W_{\mathrm{th}}$. These results are validated and are in good agreement with numerical simulations over the entire threshold range when including the experimental uncertainty. For both ramp durations $\langle W\rangle_{\mathcal{T}}-\langle\Delta F\rangle_{\mathcal{T}}$ is negative at small $W_{\text {th }}$, defying the conventional second law but is yet in agreement with Eq. (1) within experimental errors. We find that the faster is the protocol, the more negative $\langle W\rangle_{\mathcal{T}}-\langle\Delta F\rangle_{\mathcal{T}}$ becomes, which can be understood as a consequence of the irreversibility (and hence $\langle\delta\rangle_{\mathcal{T}}$ ) associated with the ramp driving speed. For large values of $W_{\text {th }}$, almost all trajectories are stopped at $\tau$ and the conventional second law is recovered, as $\langle\delta\rangle_{\mathcal{T}}$ becomes small. Furthermore, Figs. 3(b) and 3(e) report the exponential averages $\left\langle e^{-\beta(W-\Delta F)}\right\rangle_{\mathcal{T}}$ and $\left\langle e^{-\beta(W-\Delta F)-\delta}\right\rangle_{\mathcal{T}}$ evaluated at the stopping times. Notably, the conventional work fluctuation theorem $\left\langle e^{-\beta(W-\Delta F)}\right\rangle_{\mathcal{T}}=1$ only holds for large $W_{\text {th }}$, while for small $W_{\text {th }},\left\langle e^{-\beta(W-\Delta F)}\right\rangle_{\mathcal{T}}$ is significantly greater than one within experimental errors. On the other hand, we obtain an excellent agreement (with accuracy $299.5 \%$ ) of our fluctuation relation (3) for all values of $W_{\text {th }}$ and both ramp speeds. To gain further insights, in Figs. 3(c) and 3(f) we show histograms of the stopping times $\mathcal{T}$ and the value of the work at the stopping time $W(\mathcal{T})$. For small thresholds we observe that the distribution of $\mathcal{T}$ is broad and includes stopping events that take place at short times $\mathcal{T} \lesssim \Gamma_{d}^{-1}$ [Fig. 3(c), top panel]. Its corresponding distribution of $W(\mathcal{T})$ [Fig. 3(f), top panel] has a peak at $W_{\text {th }}$ arising from trajectories stopped before $\tau$ and a tail $W(\mathcal{T})<\langle\Delta F\rangle_{\mathcal{T}}$ from trajectories ending at the end of the protocol. By increasing the threshold value [Figs. 3(c) and 3(f), middle panels] we reduce the number of trajectories that stop before $\tau$ hence the distribution of $\mathcal{T}$ becomes narrower [Fig. 3(c), bottom panel]. This effect is accompanied by a broadening of the $W(\mathcal{T})$ distribution recovering a Gaussian-like shape with mean above the free energy change for large enough $W_{\text {th }}$ (i.e., typically far outside the standard fluctuation interval of $W$ ), Fig. 3(f) bottom panel.

Quantum gambling.-The gambling demon can also be extended to the quantum realm by considering quantum jump trajectories [45]. Here, the pure state of the system $|\psi(t)\rangle$ follows stochastic evolution conditioned on the 

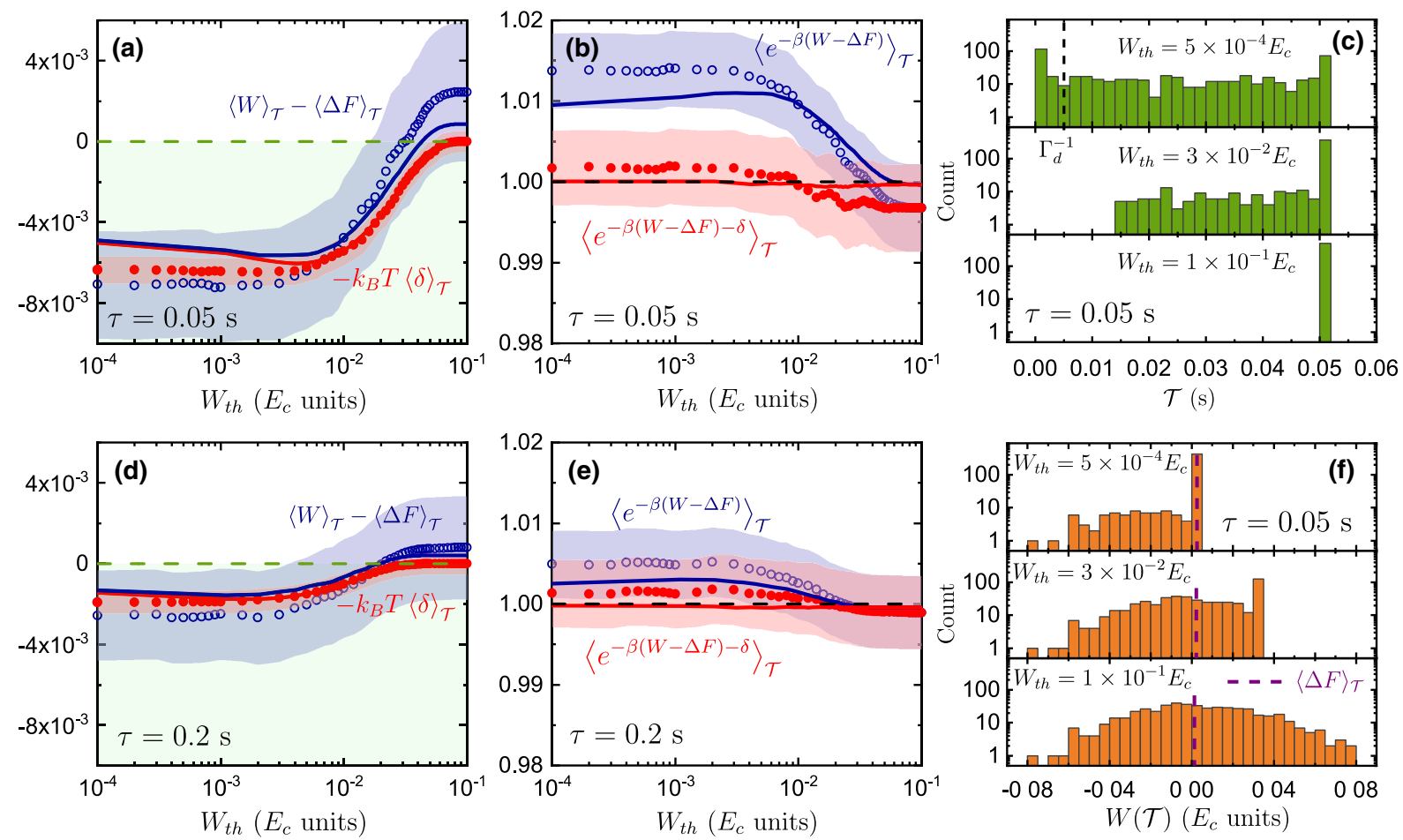

FIG. 3. Dissipated work $\langle W\rangle_{\mathcal{T}}-\langle\Delta F\rangle_{\mathcal{T}}$ (blue) and stochastic indistinguishability at stopping times (red) $-k_{B} T\langle\delta(\mathcal{T})\rangle($ dots: experimental data; solid lines: simulation) in charging energy $E_{c}=109 \mu \mathrm{eV}$ units averaged over many realizations for protocol durations $\tau=0.05 \mathrm{~s}$ (a) and $\tau=0.2 \mathrm{~s}$ (d) as a function of work threshold values. (b),(e) Test of the generalized work fluctuation relation and of Eq. (3) (dots: experimental data; solid lines: simulation) for $\tau=0.05 \mathrm{~s}$ (b) and $\tau=0.2 \mathrm{~s} \mathrm{(e).} \mathrm{(c),(f)} \mathrm{Histograms} \mathrm{of} \mathrm{stopping} \mathrm{times} \mathcal{T}$ (c) and corresponding work values $W(\mathcal{T})(\mathrm{f})$ for a ramp time $\tau=0.05 \mathrm{~s}$ for work thresholds $W_{\text {th }}=5 \times 10^{-4}, 3 \times 10^{-2}$ and $10^{-1} E_{c}$. The total uncertainty is shown by shadowed areas; it is the combination of the statistical uncertainty and error on temperature (about $10 \%$ ).

measurement outcomes generated by the continuous monitoring of the environment [46-48].

In this case, we derive the following quantum stoppingtime work fluctuation relation

$$
\left\langle e^{-\beta[W-\Delta F]-\delta_{q}+\Delta S_{\text {unc }}}\right\rangle_{\mathcal{T}}=1,
$$

where again $W$ and $\Delta F$ are, respectively, the work performed and free energy change during trajectories stopped at $\mathcal{T}$ [29]. The term $\delta_{q}(t) \equiv \ln \langle\psi(t)|\rho(t)| \psi(t)\rangle-$ $\ln \left\langle\psi(t)\left|\Theta^{\dagger} \tilde{\rho}(\tau-t) \Theta\right| \psi(t)\right\rangle$ is the quantum analog of Eq. (2), $\rho$ and $\tilde{\rho}$ being the density operators in the forward and backward process respectively, and $\Theta$ the time-reversal operator in quantum mechanics. As before, time-inversion at time $\tau$ implies $\delta_{q}(\tau)=0$. The key difference of the quantum fluctuation relation (4) with respect to its classical counterpart in Eq. (3) is the appearance of a genuine entropic term associated to quantum measurements, namely the "uncertainty" entropy production

$$
\Delta S_{\text {unc }}(\mathcal{T})=-\ln \left(\frac{\langle n(\mathcal{T})|\rho(\mathcal{T})| n(\mathcal{T}\rangle)}{\langle\psi(\mathcal{T})|\rho(\mathcal{T})| \psi(\mathcal{T})\rangle}\right)
$$

This quantity measures how much more surprising is a particular eigenstate $|n(t)\rangle$ of $\rho(t)$ with respect to the stochastic wave function $|\psi(t)\rangle$, as characterized by the logarithm of the Uhlman fidelity, $\langle\psi(t)|\rho(t)| \psi(t)\rangle$ [20]. In general, $|\psi(t)\rangle$ can be an arbitrary superposition of the instantaneous eigenstates $|n(t)\rangle$. In the classical limit the stochastic evolution of $|\psi(t)\rangle$ is given by jumps between energy levels and thus $|\psi(\mathcal{T})\rangle=|n(\mathcal{T})\rangle$. Consequently, $\Delta S_{\text {unc }}(\mathcal{T})=0$ in Eq. (5) and $\delta_{q}(\mathcal{T})=\delta(\mathcal{T})$ for any $\mathcal{T}$, thus recovering Eq. (3) in the classical limit. The corresponding stopping-time second-law inequality for quantum systems reads $\langle W\rangle_{\mathcal{T}}-\langle\Delta F\rangle_{\mathcal{T}} \geq-k_{B} T\left(\left\langle\delta_{q}\right\rangle_{\mathcal{T}}-\left\langle\Delta S_{\text {unc }}\right\rangle_{\mathcal{T}}\right)$, where $\left\langle\Delta S_{\text {unc }}\right\rangle_{\mathcal{T}}$ modifies the entropic balance. Even if $\left\langle\Delta S_{\text {unc }}\right\rangle \geq$ 0 for any fixed time $t \leq \tau$, the average over stopped trajectories $\left\langle\Delta S_{\text {unc }}\right\rangle_{\mathcal{T}}$ may be either positive or negative depending on the selected gambling strategy. Therefore, the quantum fluctuations induced by measurements may act either as an entropy source or as an entropy sink.

Conclusions.-We have introduced and illustrated the stochastic thermodynamics of gambling demons, i.e., driven nonequilibrium processes that are stopped at stochastic times following a prescribed criterion. Our results generalize the second law to arbitrary stopping ("gambling") strategies for classical and quantum systems driven out of equilibrium. Even though all finite-time horizon gambling strategies fulfill the stopping-time fluctuation relation (3) and the inequality (1), not all guarantee average work extraction above the average nonequilibrium free 
energy change. Such "negative dissipation" requires the usage of gambling strategies in a sufficiently irreversible process: stopping the dynamics at stochastic times with a suitable gambling strategy, and a time-asymmetric driving protocol. This contrasts with heat and information engines which achieve maximal work extraction in the quasistatic reversible limit $[49,50]$.

Our relations are fundamentally different to the generalized second law with feedback $\langle W\rangle-\langle\Delta F\rangle \geq-k_{B} T I$, where $I$ is the information acquired by a feedback controller from the system in a fixed-time protocol $[3,4]$, or at stochastic times [9]. The information used to implement a gambling strategy can be estimated assuming periodic measurements every sampling time $\Delta t$, each providing at least a bit of information, corresponding to "stop" or "don't stop" the trajectory. In the small sampling time limit, these measurements generate sequences of $N \sim \mathcal{T} / \Delta t+1$ bits per trajectory. Erasing these bits would have an energetic cost that becomes infinitely large in the continuous measurement limit $\Delta t \rightarrow 0$ [9]. Our results show that gambling demons are, nevertheless, constrained by the bound in Eq. (1), which is tighter than an extension of the second law with feedback at stopping times. In the experiment reported here we indeed obtain $k_{B} T\langle\delta\rangle_{\mathcal{T}} \sim 7.8 \times 10^{-3} k_{B} T \ll$ $k T \ln 2$, but faster protocols are expected to achieve larger values of $\langle\delta\rangle_{\mathcal{T}}$. It would be interesting in the future to further investigate the interplay between our fluctuation relations and information acquisition, as well as with recent stopping-time uncertainty relations [51], and speed limits [52]. Applications to experimental quantum devices $[53,54]$ may allow us to exploit quantum superpositions to enhance work extraction beyond the classical limits. Finally, it would be interesting to explore optimization of stopping strategies using knowledge in quantitative finance (e.g., option pricing, arbitrage, etc.) and gambling [55,56] such as Parrondo games [57].

We acknowledge fruitful discussions with Christopher Jarzynski. G. M. acknowledges funding from the European Union's Horizon 2020 research and innovation programme under the Marie Skłodowska-Curie Grant Agreement No. 801110 and the Austrian Federal Ministry of Education, Science and Research (BMBWF). R. F. research has been conducted within the framework of the Trieste Institute for Theoretical Quantum Technologies (TQT). This work was funded through Academy of Finland Grant No. 312057 and from the European Unions Horizon 2020 research and innovation programme under the European Research Council (ERC) programme.

*gonzalo.manzano@oeaw.ac.at †edgar@ictp.it

[1] A. Rex and H.S. Leff, Maxwell's Demon 2: Entropy, Classical and Quantum Information, Computing (Taylor and Francis, London, 2002).
[2] K. Maruyama, F. Nori, and V. Vedral, Colloquium: The physics of Maxwell's demon and information, Rev. Mod. Phys. 81, 1 (2009).

[3] T. Sagawa and M. Ueda, Second Law of Thermodynamics with Discrete Quantum Feedback Control, Phys. Rev. Lett. 100, 080403 (2008).

[4] T. Sagawa and M. Ueda, Generalized Jarzynski Equality Under Nonequilibrium Feedback Control, Phys. Rev. Lett. 104, 090602 (2010).

[5] L. Del Rio, J. Åberg, R. Renner, O. Dahlsten, and V. Vedral, The thermodynamic meaning of negative entropy, Nature (London) 474, 61 (2011).

[6] J. M. R. Parrondo, J. M. Horowitz, and T. Sagawa, Thermodynamics of information, Nat. Phys. 11, 131 (2015).

[7] E. Lutz and S. Ciliberto, Information: From Maxwell's demon to Landauer's eraser, Phys. Today 68, No. 9, 30 (2015).

[8] M. Gavrilov and J. Bechhoefer, Erasure without Work in an Asymmetric Double-Well Potential, Phys. Rev. Lett. 117, 200601 (2016).

[9] M. Ribezzi-Crivellari and F. Ritort, Large work extraction and the Landauer limit in a continuous Maxwell demon, Nat. Phys. 15, 660 (2019).

[10] P. A. Camati, J. P. S Peterson, T. B. Batalhao, K. Micadei, A. M. Souza, R. S. Sarthour, I. S. Oliveira, and R. M. Serra, Experimental Rectification of Entropy Production by Maxwell's Demon in a Quantum System, Phys. Rev. Lett. 117, 240502 (2016).

[11] N. Cottet, S. Jezouin, L. Bretheau, P. Campagne-Ibarcq, Q. Ficheux, J. Anders, A. Auffèves, R. Azouit, P. Rouchon, and B. Huard, Observing a quantum Maxwell demon at work, Proc. Natl. Acad. Sci. U.S.A. 114, 7561 (2017).

[12] D. Williams, Probability with Martingales (Cambridge University Press, Cambridge, England, 1991).

[13] S. Pliska, Introduction to Mathematical Finance (Blackwell Publishers, Oxford, 1997).

[14] R. Chetrite and S. Gupta, Two refreshing views of fluctuation theorems through kinematics elements and exponential martingale, J. Stat. Phys. 143, 543 (2011).

[15] I. Neri, É. Roldán, and F. Jülicher, Statistics of Infima and Stopping Times of Entropy Production and Applications to Active Molecular Processes, Phys. Rev. X 7, 011019 (2017).

[16] C. Moslonka and K. Sekimoto, Memory through a hidden Martingale process in progressive quenching, Phys. Rev. E 101, 062139 (2020).

[17] H. Ge, C. Jia, and X. Jin, Martingale structure for general thermodynamic functionals of diffusion processes under second-order averaging, arXiv:1811.04529.

[18] Y.-J. Yang and H. Qian, Unified formalism for entropy production and fluctuation relations, Phys. Rev. E 101, 022129 (2020).

[19] R. Chétrite, S. Gupta, I. Neri, and É. Roldán, Martingale theory for housekeeping heat, Europhys. Lett. 124, 60006 (2019).

[20] G. Manzano, R. Fazio, and É. Roldán, Quantum Martingale Theory and Entropy Production, Phys. Rev. Lett. 122, 220602 (2019).

[21] I. Neri, É. Roldán, S. Pigolotti, and F. Jülicher, Integral fluctuation relations for entropy production at stopping times, J. Stat. Mech. (2019) 104006. 
[22] I. Neri, Second Law of Thermodynamics at Stopping Times, Phys. Rev. Lett. 124, 040601 (2020).

[23] K. Sekimoto, Stochastic Energetics (Springer, New York, 2010), Vol. 799.

[24] U. Seifert, Stochastic thermodynamics, fluctuation theorems and molecular machines, Rep. Prog. Phys. 75, 126001 (2012).

[25] C. Jarzynski, Equalities and inequalities: Irreversibility and the second law of thermodynamics at the nanoscale, Annu. Rev. Condens. Matter Phys. 2, 329 (2011).

[26] C. Jarzynski, Nonequilibrium Equality for Free Energy Differences, Phys. Rev. Lett. 78, 2690 (1997).

[27] U. Seifert, Entropy Production Along a Stochastic Trajectory and an Integral Fluctuation Theorem, Phys. Rev. Lett. 95, 040602 (2005).

[28] Here, $\tilde{x}= \pm x$ represents the parity of the variable $x$ under time reversal.

[29] See Supplemental Material at http://link.aps.org/ supplemental/10.1103/PhysRevLett.126.080603 for theoretical and experimental details, as well as proofs of the main results, which includes Refs. [30-41].

[30] G. Lindblad, On the generators of quantum dynamical semigroups, Commun. Math. Phys. 48, 119 (1976).

[31] J. M. Horowitz and J. M. R. Parrondo, Entropy production along nonequilibrium quantum jump trajectories, New J. Phys. 15, 085028 (2013).

[32] B. Leggio, A. Napoli, A. Messina, and H.-P. Breuer, Entropy production and information fluctuations along quantum trajectories, Phys. Rev. A 88, 042111 (2013).

[33] M. Campisi, J. P. Pekola, and R. Fazio, Nonequilibrium fluctuations in quantum heat engines: Theory, example, and possible solid state experiments, New J. Phys. 17, 035012 (2015).

[34] G. Manzano, J. M. Horowitz, and J. M. R. Parrondo, Nonequilibrium potential and fluctuation theorems for quantum maps, Phys. Rev. E 92, 032129 (2015).

[35] Z. Gong, Y. Ashida, and M. Ueda, Quantum-trajectory thermodynamics with discrete feedback control, Phys. Rev. A 94, 012107 (2016).

[36] F. Liu and J. Xi, Characteristic functions based on a quantum jump trajectory, Phys. Rev. E 94, 062133 (2016).

[37] C. Elouard, D. A. Herrera-Martí, M. Clusel, and A. Auffèves, The role of quantum measurement in stochastic thermodynamics, npj Quantum Inf. 3, 9 (2017).

[38] B. Karimi and J. P. Pekola, Quantum Trajectory Analysis of Single Microwave Photon Detection by Nanocalorimetry, Phys. Rev. Lett. 124, 170601 (2020).

[39] R. Kawai, J. M. R. Parrondo, and C. Van den Broeck, Dissipation: The Phase-Space Perspective, Phys. Rev. Lett. 98, 080602 (2007).

[40] T. Sagawa, Second Law-Like Inequalities with Quantum Relative Entropy: An Introduction in Lectures on Quantum Computing, Thermodynamics and Statistical Physics, edited by M. Nakahara and S. Tanaka, Kinki University Series on Quantum Computing (World Scientific, Singapore, 2012).

[41] J. Doob, Stochastic Processes (John Wiley and Sons, New York, 1953).
[42] Here, we refer to apparent "second-law violations" since the second law can be restored by considering the erasure of the information acquired to implement the gambling strategy, as in other versions of Maxwell's demon.

[43] O. Maillet, P. A. Erdman, V. Cavina, B. Bhandari, E. T. Mannila, J. T. Peltonen, A. Mari, F. Taddei, C. Jarzynski, V. Giovannetti, and J. P. Pekola, Optimal Probabilistic Work Extraction Beyond the Free Energy Difference with a Single-Electron Device, Phys. Rev. Lett. 122, 150604 (2019).

[44] J. P. Pekola and I. M. Khaymovich, Thermodynamics in single-electron circuits and superconducting qubits, Annu. Rev. Condens. Matter Phys. 10, 193 (2019).

[45] H. M. Wiseman and G. J. Milburn, Quantum Measurement and Control (Cambridge University Press, Cambridge, England, 2009).

[46] J. M. Horowitz, Quantum-trajectory approach to the stochastic thermodynamics of a forced harmonic oscillator, Phys. Rev. E 85, 031110 (2012).

[47] F. W. J. Hekking and J. P. Pekola, Quantum Jump Approach for Work and Dissipation in a Two-Level System, Phys. Rev. Lett. 111, 093602 (2013).

[48] G. Manzano, J. M. Horowitz, and J. M. R. Parrondo, Quantum Fluctuation Theorems for Arbitrary Environments: Adiabatic and Nonadiabatic Entropy Production, Phys. Rev. X 8, 031037 (2018).

[49] I. A. Martínez, É. Roldán, L. Dinis, D. Petrov, J. M. Parrondo, and R. A. Rica, Brownian Carnot engine, Nat. Phys. 12, 67 (2016).

[50] J. M. Horowitz and J. M. R. Parrondo, Thermodynamic reversibility in feedback processes, Europhys. Lett. 95, 10005 (2011).

[51] G. Falasco and M. Esposito, Dissipation-Time Uncertainty Relation, Phys. Rev. Lett. 125, 120604 (2020).

[52] N. Shiraishi, K. Funo, and K. Saito, Speed Limit for Classical Stochastic Processes, Phys. Rev. Lett. 121, 070601 (2018).

[53] Z. K. Minev, S. O. Mundhada, S. Shankar, P. Reinhold, R. Gutiérrez-Jáuregui, R. J. Schoelkopf, M. Mirrahimi, H. J. Carmichael, and M.H. Devoret, To catch and reverse a quantum jump mid-flight, Nature (London) 570, 200 (2019).

[54] K. W. Murch, S. Weber, C. Macklin, and I. Siddiqi, Observing single quantum trajectories of a superconducting quantum bit, Nature (London) 502, 211 (2013).

[55] L. Dinis, J. Unterberger, and D. Lacoste, Phase transitions in optimal strategies for betting, Eur. Phys. Lett. 131, 60005 (2020).

[56] S. Ito, Backward transfer entropy: Informational measure for detecting hidden Markov models and its interpretations in thermodynamics, gambling and causality, Sci. Rep. 6, 36831 (2016).

[57] G. P. Harmer and D. Abbott, Losing strategies can win by Parrondo's paradox, Nature (London) 402, 864 (1999). 